\title{
Learning probabilistic models of connectivity from multiple spike train data
}

\author{
Debprakash Patnaik ${ }^{1 *}$, Srivatsan Laxman², Naren Ramakrishnan ${ }^{1}$ \\ From Nineteenth Annual Computational Neuroscience Meeting: CNS*2010 \\ San Antonio, TX, USA. 24-30 July 2010
}

Neuronal circuits or cell assemblies carry out brain function through complex coordinated firing patterns [1]. Inferring topology of neuronal circuits from simultaneously recorded spike train data is a challenging problem in neuroscience. In this work we present a new class of dynamic Bayesian networks to infer polysynaptic excitatory connectivity between spiking cortical neurons [2]. The emphasis on excitatory networks allows us to learn connectivity models by exploiting fast data mining algorithms. Specifically, we show that frequent episodes help identify nodes with high mutual information relationships and can be summarized into a dynamic Bayesian network (DBN).

We model the spike train data as binary random variables and learn high mutual information parent sets of neurons that excite the spiking of down-stream neurons at variable delays. Thus we can express the probability of spiking of each neuron conditioned on the activity of a subset of relevant neurons in recent past (or history window). We formally establish a connection between efficient frequent episode mining algorithms (used to indentify frequently repeating patterns of spiking activity [3]) and learning probabilistic models for excitatory connections. This framework is depicted in Figure 1.

We demonstrate the effectiveness of our method in discovering connectivity information on synthetic and real datasets. Our synthetic data generation models each neuron as an inhomogeneous Poisson process whose firing rate is modulated by the input received by the neuron in recent past. The network inter-connect allows us to model complex higher-order interactions. We also demonstrate the application of our method on multi-electrode arrays recordings from dissociated cortical cultures gathered by Steve Potter's laboratory at Georgia Tech [4].

\section{Conclusion}

Existing data analysis tools like cross-correlograms, JPSTH and PCA do not scale well as we look at several

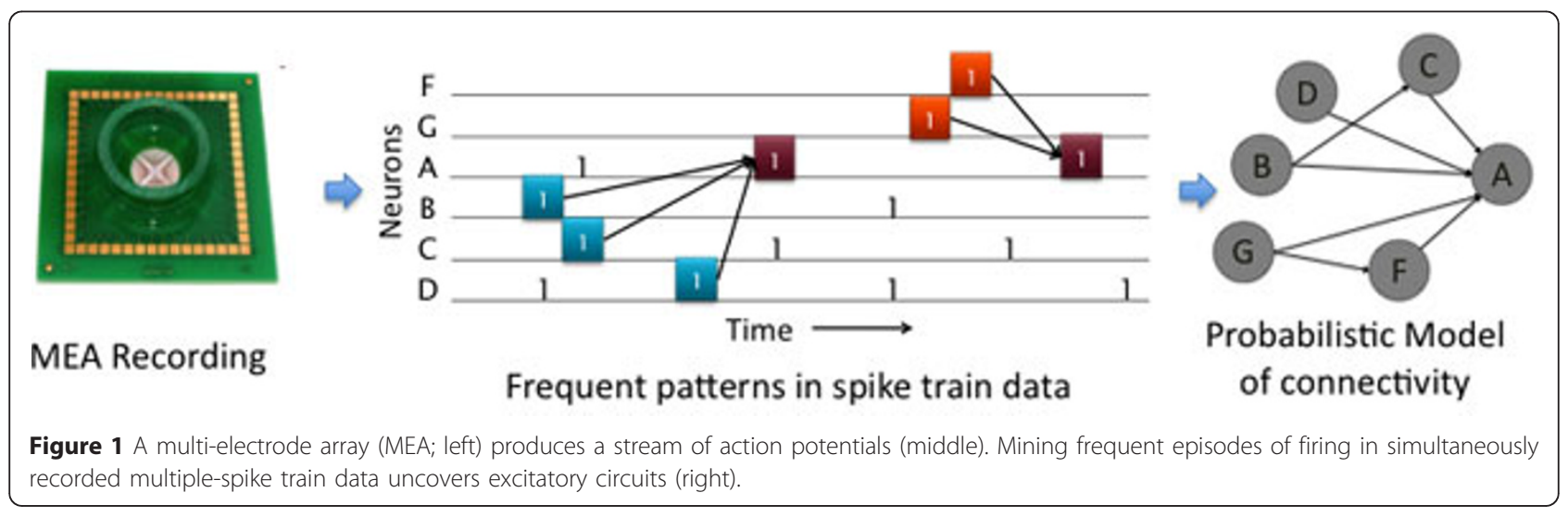

\footnotetext{
* Correspondence: patnaik@vt.edu

${ }^{1}$ Computer Science Department, Virginia Polytechnic Institute and State University, Blacksburg, VA 24060, USA
} 

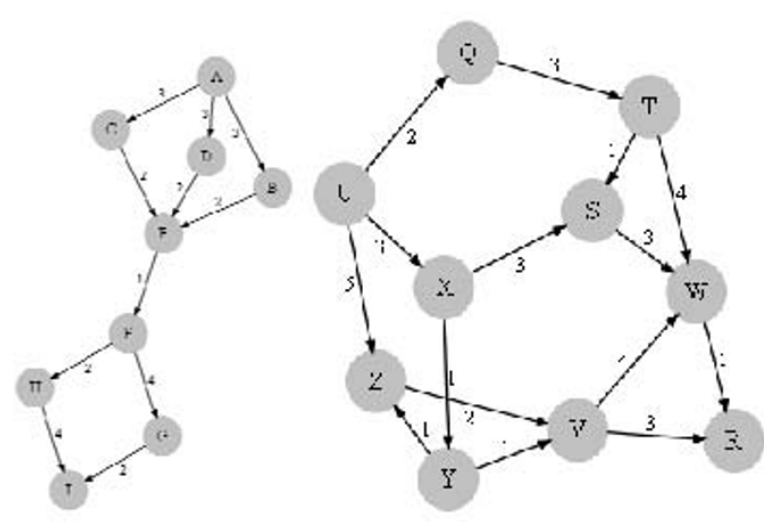

Figure 2 Dynamic Bayesian Network models for Syn-fire chains (left) and Polychronous circuits (right).

neurons at a time. Our approach provides an efficient and formal basis for learning probabilistic models from observed spike train data. Several types of network dynamics like syn-fire chains, polychrony [5] etc. that neuronal networks are known to exhibit can be modeled as excitatory networks and hence their putative structure can be learnt using our method (as illustrated in Figure 2). Our proposed approach also scales very well to large data sizes as it marries fast data mining style algorithms with formal model learning.

\section{Author details}

${ }^{1}$ Computer Science Department, Virginia Polytechnic Institute and State University, Blacksburg, VA 24060, USA. ${ }^{2}$ Microsoft Research, Bangalore,

Karnataka 560080, India.

Published: 20 July 2010

\section{References}

1. Abeles $M$, Gerstein GL: Detecting spatiotemporal firing patterns among simultaneously recorded single neurons. J Neurophysiol 1988, 60(3):909-924.

2. Patnaik D, Laxman S, Ramakrishnan N: Discovering excitatory networks from discrete event streams with applications to neuronal spike train analysis. Proc. of IEEE Intl. Conf. Data Mining, ICDM 2009.

3. Patnaik D, Sastry PS, Unnikrishnan KP: Inferring neuronal network connectivity from spike data: A temporal data mining approach. Scientific Programming 2007, 16(1):49-77.

4. Wagenaar DA, Pine J, Potter SM: An extremely rich repertoire of bursting patterns during the development of cortical cultures. BMC Neuroscience 2006.

5. Izhikevich EM: Polychronization: Computation with spikes. Neural Comput. 2006, 18(2):245-282.

doi:10.1186/1471-2202-11-S1-P171

Cite this article as: Patnaik et al:: Learning probabilistic models of connectivity from multiple spike train data. BMC Neuroscience 2010 11(Suppl 1):P171.

\section{Submit your next manuscript to BioMed Central and take full advantage of:}

- Convenient online submission

- Thorough peer review

- No space constraints or color figure charges

- Immediate publication on acceptance

- Inclusion in PubMed, CAS, Scopus and Google Scholar

- Research which is freely available for redistribution

Submit your manuscript at www.biomedcentral.com/submit 